\title{
Adaptive Threshold Based Relay Selection for Minimum Feedback
}

\author{
(Invited Paper) \\ Sung Chul Park and Dong In Kim \\ School of Information and Communication Engineering, Sungkyunkwan University (SKKU) \\ Suwon 440-746, Korea \\ scpark94@samsung.com, dongin@skku.edu
}

\begin{abstract}
To enable relay selection in multiple relay network, using an average channel gain is not enough to achieve acceptable performance, but using an instantaneous gain causes undue system overhead due to feedback. In this paper, we propose adaptive threshold based relay selection scheme for minimum feedback to avoid system overhead, where a relay reports if its relay-destination (R-D) instantaneous channel gain is above threshold. A source selects the best relay among those which has the highest source-relay (S-R) instantaneous channel gain, and adjusts adaptive threshold according to an operating signalto-noise ratio. Exact and upper bound on the symbol error rate are derived for $M$-PSK signaling, and it is shown that the proposed scheme yields acceptable performance in operating range, though full diversity order is not achieved. The theoretical results presented here are validated through simulation.
\end{abstract}

\section{INTRODUCTION}

Diversity method is one of the key solutions to overcome multipath fading in wireless network area. Multiple antennas can be used to achieve diversity, but due to small size and hardware complexity, it is difficult to deploy more than one antenna in wireless device. Cooperative communication for wireless networks is a promising solution which can achieve spatial diversity without installing multiple antennas. In cooperative communications, relay nodes act as virtual antenna array to help source forward its information to destination.

Various cooperative communication methods have been proposed and analyzed. In [1] - [3], Decode-and-Forward (DF) and Amplify-and-Forward (AF) were proposed to achieve spatial diversity. In [4], authors analyzed the performance of two-hop relay communication by the harmonic mean of two independent exponential distributions. In [5], they provided the symbol error rate (SER) expression and an optimum power allocation scheme for DF system.

Conventional relay network with $N$ relays requires $\mathrm{N}+1$ time slots per packet. In the first phase, a source broadcasts data to a destination and multiple relays. In the $i$-th phase, a relay combines all signals coming from the source and relays, and forwards if decode correctly. Consequently it uses $\mathrm{N}+1$ time slots per packet, causing degradation of spectral efficiency. For this reason, the best relay selection method was proposed for efficient use of spectrum. In the best relay selection method, it requires 2 time slots per packet and can achieve full diversity order. In [6], the best relay is the one which has the highest harmonic mean of $S-R$ (sourcerelay) and $R-D$ (relay-destination) channel gains. Here, authors proposed a method to improve the symbol per channel use
(SPCU) by comparing the best relay's harmonic mean with its $S-D$ (source-destination) channel gain weighted by a factor of cooperation threshold. If the harmonic mean is higher than the weighted $S-D$ channel gain, it adopts cooperative transmission, otherwise direct transmission.

The problem of the best relay selection is that it requires global channel state information (CSI) to determine the best relay. If the system is operated in time-division duplex (TDD), a source can acquire information about instantaneous $S-D$ and $S-R$ channel gains, while a relay information about $S$ $R$ and $R-D$ channel gains. In addition, if the source obtains the $R-D$ channel gain, it can achieve full diversity order, but requires feedback from the relay and causes cooperation overhead. Without knowing this $R-D$ channel gain, the system performance is down to the second order diversity, which is same as a single relay system even if its average channel gain is available at the source. The average channel gain does not provide the time-selective information, and it is less likely to select the best relay in most of time.

In this paper, we propose Adaptive Threshold based Relay Selection (ATRS) for DF system, which aims to minimize feedback with acceptable performance. The ATRS scheme generates only one-bit information for feedback, and adaptive threshold for acceptable performance is optimized by referring to an operating signal-to-noise ratio (SNR). The ATRS scheme cannot achieve full diversity order in high SNR region, but it provides acceptable performance in most practical SNR range. Simplicity of the ATRS scheme facilitates easy approach to practical radio systems.

The rest of the paper is organized as follows. Section II presents the system model for multiple relay DF cooperative wireless network, along with the ATRS scheme for the best relay selection. In Section III, we derive exact and upper bound on the symbol error rate (SER) for $M$-ary phase-shift keying ( $M$-PSK) signaling. Section IV presents numerical and simulation results to validate the performance of the ATRS scheme. Finally, concluding remarks are given in Section V.

\section{SySTEM MODEL}

We consider multiple relay DF cooperative network shown in Fig. 1. $S$ is source node, $D$ is destination node, $R_{i}$ is relay node, and there exist $\mathrm{N}$ relays. The best relay selection based DF protocol consists of two time slots. In the first phase, a source broadcasts data to the best relay and a destination. The 


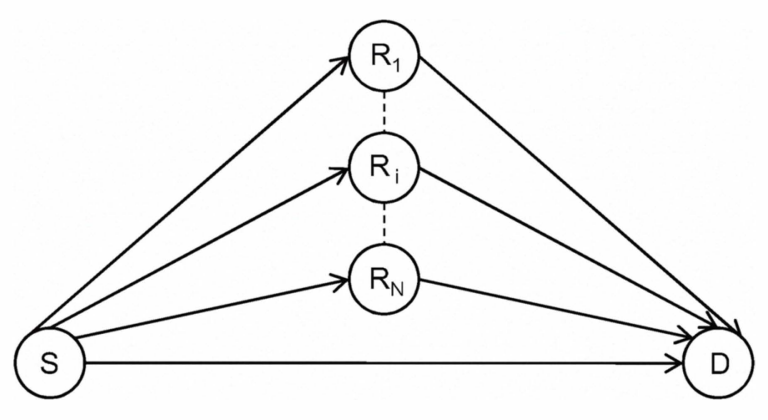

Fig. 1. Multiple relay network model with $N$ relays.

received signals at their respective nodes are expressed as

$$
\begin{aligned}
y_{s, d} & =\sqrt{P_{1}} h_{s, d} x+n_{s, d} \\
y_{s, r_{i}} & =\sqrt{P_{1}} h_{s, r_{i}} x+n_{s, r_{i}}
\end{aligned}
$$

where $P_{1}$ is the transmission power at a source, $x$ is the transmitted symbol with unit power, $h_{s, d}$ and $h_{s, r_{i}}$ are the channel gains of $S-D$ and $S-R_{i}$ links, $n_{s, d}$ and $n_{s, r_{i}}$ are additive white Gaussian noises (AWGNs) over the links.

In the second phase, the best relay forwards data to the destination if decode correctly, otherwise the relay remains idle. The received signal at the destination is expressed as

$$
y_{r_{i}, d}=\sqrt{\tilde{P}_{2}} h_{r_{i}, d} x+n_{r_{i}, d}
$$

where $x$ is the transmitted symbol from the best relay, $h_{r_{i}, d}$ is the channel gain of $R_{i}-D$ link, and $n_{r_{i}, d}$ is AWGN over the link. Note that $\tilde{P}_{2}=P_{2}$ if the best relay decodes correctly and otherwise $\tilde{P}_{2}=0$. The system has the total power constraint of $P=P_{1}+P_{2}$, where $P$ is the maximum transmission power available. We assume maximal-ratio combining (MRC) for combining the signals from both source and relay to destination. We also assume flat quasi-static channel so that channel gains remain constant over the time slot and change independently from slot to slot.

In the relay selection scheme, we assume TDD mode where $h_{s, d}$ and $h_{s, r_{i}}$ are known at a source, and $h_{r_{i}, d}$ known at a relay, but $h_{r_{i}, d}$ must be fedback to the source by the relay that causes system overhead. Instead, the proposed ATRS scheme employs a threshold method that requires 'one-bit information' to minimize feedback. The scheme works as follows. In the first step, the relay $R_{i}$ notifies the source of being "available" if its $R_{i}$-D channel gain $h_{r_{i}, d}$ exceeds threshold

$$
\beta_{r_{i}, d} \geq \beta_{t h} \longrightarrow \text { available link }
$$

where $\beta_{x, y}=\left|h_{x, y}\right|^{2}$ and $\beta_{t h}$ is the threshold. Such relays whose $R-D$ links are available, are considered as candidates for the best relay. In the second step, the source selects the best relay among them which has the maximum $\left|h_{s, r_{i}}\right|$ as

$$
\max _{i}\left\{\beta_{s, r_{1}}, \ldots, \beta_{s, r_{k}}\right\} \quad \text { for } k \text { candidates. }
$$

In case there exists no candidate $(k=0)$, the source randomly chooses a relay among $N$ relays. One hand is to use direct transmission with all available power $P$ in the first phase, but this method is shown to perform worse than the random relay selection. This means that combining diversity yields better performance even though all $R-D$ channel gains are not sufficiently enough. The other hand is to choose a relay with highest $S-R_{i}$ channel gain among $N$ relays. But it performs similar to the random relay selection, so that searching for the highest $S-R_{i}$ is meaningless in no candidate case.

The channel gains $h_{s, d}, h_{s, r_{i}}$, and $h_{r_{i}, d}$ are zero-mean complex Gaussian random variables with variances $\delta_{s, d}^{2}, \delta_{s, r_{i}}^{2}$, and $\delta_{r_{i}, d}^{2}$, respectively. Hence, $\left|h_{x, y}\right|$ is Rayleigh distributed and $\left|h_{x, y}\right|^{2}$ is exponentially distributed with mean $\delta_{x, y}^{2} . n_{s, d}$, $n_{s, r_{i}}$, and $n_{r i, d}$ are zero-mean complex Gaussian random variables with variance $\sigma_{n}^{2}$.

\section{Performance Analysis}

We analyze the SER performance of the proposed ATRS scheme for $M$-PSK signaling. We formulate an exact closedform SER expression, and then derive its upper bound for a simple calculation of adaptive threshold.

First, the instantaneous SNRs of $S-D$ and $S-R_{i}$ links are defined as

$$
\gamma_{s, d}=\frac{P_{1} \beta_{s, d}}{\sigma_{n}^{2}}, \quad \gamma_{s, r_{i}}=\frac{P_{1} \beta_{s, r_{i}}}{\sigma_{n}^{2}} .
$$

For cooperation diversity, the system employs MRC at the destination whose output signal is of the form

$$
y=\frac{\sqrt{P_{1}} h_{s, d}^{*} y_{s, d}+\sqrt{P_{2}} h_{r_{i}, d}^{*} y_{r_{i}, d}}{\sigma_{n}^{2}},
$$

and the instantaneous SNR of MRC output can be evaluated as

$$
\gamma_{s, r_{i}, d}=\frac{P_{1} \beta_{s, d}+P_{2} \beta_{r_{i}, d}}{\sigma_{n}^{2}} .
$$

The SER formula for $M$-PSK signaling is given by [7]

$$
P_{e}(\gamma)=\frac{1}{\pi} \int_{0}^{(M-1) \pi / M} \exp \left(-\frac{b \gamma}{\sin ^{2} \theta}\right) d \theta
$$

where $b=\sin ^{2}(\pi / M)$ and $M$ is the modulation order of PSK. The probability of having a candidate relay subset of size $k$ is

$$
\operatorname{Pr}\left[R_{k}\right]=\prod_{i \in R_{k}} \operatorname{Pr}\left[\beta_{r_{i}, d} \geq \beta_{t h}\right] \prod_{i \notin R_{k}} \operatorname{Pr}\left[\beta_{r_{i}, d}<\beta_{t h}\right]
$$

where $R_{k}$ is a relay subset with k candidates (i.e., cardinality $\left|R_{k}\right|=k$ ). For simplicity, we assume that all relays are identical, such as $\delta_{s, r_{1}}^{2}=\ldots=\delta_{s, r_{N}}^{2}=\delta_{s, r}^{2}$ and $\delta_{r_{1}, d}^{2}=$ $\ldots=\delta_{r_{N}, d}^{2}=\delta_{r, d}^{2}$. Then, the problem is simplified as "How many candidate relays exist?". The probability of having $\mathrm{k}$ candidates follows the binomial distribution, which is

$$
P(K=k)=\left(\begin{array}{c}
N \\
k
\end{array}\right)\left(e^{-\beta_{t h} / \delta_{r, d}^{2}}\right)^{k}\left(1-e^{-\beta_{t h} / \delta_{r, d}^{2}}\right)^{N-k} .
$$

Since the SER is conditioned on the number of candidates $k$, the average SER can be evaluated as

$$
P_{e}=\sum_{k=0}^{N} P_{e}(k) P(K=k) \text {. }
$$


The probability density function ( $p d f)$ of each link channel gain is defined as follows. First, $S$ - $D$ link is independent of the number of candidates, and its channel gain has the exponential distribution

$$
p_{s, d}(\beta \mid K=k)=p_{s, d}(\beta)=\frac{1}{\delta_{s, d}^{2}} e^{-\beta / \delta_{s, d}^{2}} .
$$

Now we consider the ATRS scheme where there exists at least one candidate relay $(k>0)$. For $S-R$ links, a source selects a relay with maximum $S-R_{i}$ channel gain among $k$ candidates, whose cumulative distribution function $(C D F)$ is

$$
\begin{aligned}
P_{s, r_{i}}(\beta \mid K=k) & =\operatorname{Pr}\left(\beta_{s, r_{1}}<\beta, \ldots, \beta_{s, r_{k}}<\beta\right) \\
& =\left(1-e^{-\beta / \delta_{s, r}^{2}}\right)^{k}
\end{aligned}
$$

and the $p d f$ of $S-R_{i}$ channel gain is

$$
p_{s, r_{i}}(\beta \mid K=k)=\frac{k}{\delta_{s, r}^{2}} e^{-\beta / \delta_{s, r}^{2}}\left(1-e^{-\beta / \delta_{s, r}^{2}}\right)^{k-1} .
$$

At the chosen $R_{i}-D$ link, its channel gain is within $\beta_{t h} \leq \beta<$ $\infty$ due to threshold, and the $p d f$ of $R_{i}-D$ channel gain is

$$
p_{r_{i}, d}(\beta \mid K=k)=\frac{1}{\delta_{r, d}^{2}} e^{\left(\beta-\beta_{t h}\right) / \delta_{r, d}^{2}} .
$$

Next we consider the case of no relay candidate (i.e., $k=0$ ), in which case a relay is selected randomly, and the $p d f$ of $S-R_{i}$ channel gain is exponentially distributed with average $\delta_{s, r}^{2}$. Since all $R-D$ channel gains are below a given threshold $\left(0 \leq \beta<\beta_{t h}\right)$, the $p d f$ of $R_{i}-D$ channel gain is

$$
p_{r_{i}, d}(\beta \mid K=0)=\frac{1}{\delta_{r, d}^{2}} \frac{e^{-\beta / \delta_{r, d}^{2}}}{\left(1-e^{-\beta_{t h} / \delta_{r, d}^{2}}\right)}
$$

Finally, the SER performance of the DF system with ATRS can be formulated as

$$
P_{e}(k)=P_{e}\left(s, r_{i} \mid k\right) P_{e}(s, d)+\left[1-P_{e}\left(s, r_{i} \mid k\right)\right] P_{e}\left(s, r_{i}, d \mid k\right)
$$

where $P_{e}\left(s, r_{i} \mid k\right)$ denotes the decoding error at the best relay among $k$ candidates, $P_{e}(s, d)$ and $P_{e}\left(s, r_{i}, d \mid k\right)$ represent the SERs for direct and cooperative transmissions, respectively.

Now we found all parameters and the formula to derive the SER performance. Substituting the corresponding SNRs into (6), (15) and (9), and taking the expectation of each channel gains, we derive (17), (18) and (19) when $K>0$. With $K=0$, we derive (20), (21) and (22), where

$$
F_{1}(x(\theta))=\frac{1}{\pi} \int_{0}^{(M-1) \pi / M} \frac{1}{x(\theta)} d \theta .
$$

Combining (17)-(22) yields an exact form of the SER expression. We can compute the SER from the exact form, but extensive numerical calculation is required due to integration, and it may be difficult to find an optimal threshold. Hence, we obtain its upper bound for a simple calculation. Since $0 \leq \sin ^{2} \theta \leq 1$, substituting $\sin ^{2} \theta=1$, removing $i$ in (16) for high SNR, and neglecting negative terms, we derive (23).

Fig. 2 shows the adaptive threshold $\beta_{t h}$ versus operating $\mathrm{SNR} \triangleq P / \sigma_{n}^{2}$, where $\beta_{t h}$ is found by minimizing the upper bound on SER in (23). We observe that as the number of

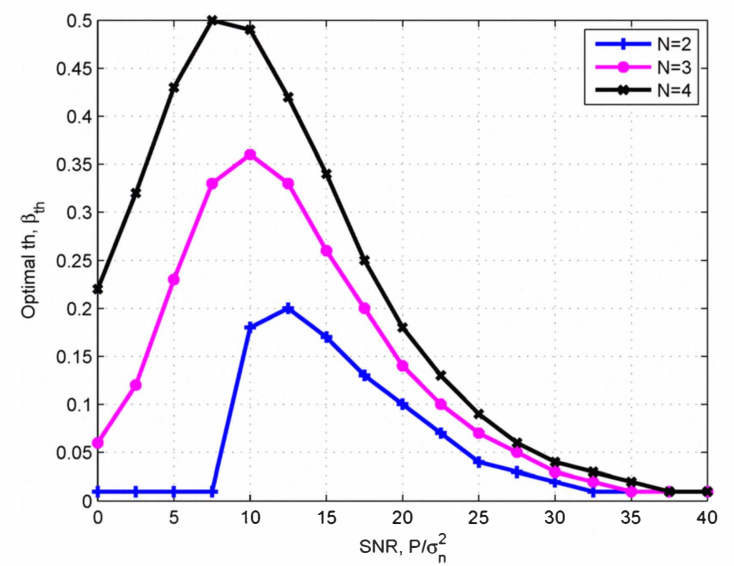

Fig. 2. Adaptive threshold $\beta_{t h}$ versus operating SNR $P / \sigma_{n}^{2}$.

relays increases, $\beta_{t h}$ also increases. Intuitively, with a large number of relays, it is more likely to have a suitable number of candidates at higher threshold, while the threshold should be lowered for a small number of relays. It is also seen that the practical operating SNR range is around 0 to $30 \mathrm{~dB}$, and an optimal threshold converges to 0 when SNR goes to extremely high. This means that all relays are candidates, and a source just finds a relay with the maximum $S-R_{i}$ channel gain. In other words, feedback is not necessary, in which case the SER is

$$
P_{e}<\left(\frac{P}{\sigma_{n}^{2}}\right)^{-2}\left(\frac{M-1}{4 M b^{2} \delta_{s, d}^{2} \delta_{r, d}^{2}}+\frac{(M-1)^{2}}{4 M^{2} b^{2} \delta_{s, d}^{2} \delta_{s, r}^{2}}\right) .
$$

Note that the diversity order is defined by

$$
d=-\lim _{S N R \rightarrow \infty} \log \left(P_{e}\right) / \log (S N R),
$$

which gives the diversity order of two in (24), but the operating SNR range here is suitable for practical radio systems. Actual performance and verification are presented in next section.

\section{RESULTS}

Simulation results are presented for the ATRS scheme to validate theoretical results. Simulation and numerical analysis are performed under equal power allocation with $P_{1}=P_{2}$, and the modulation order is $M=4$ in PSK signaling. Noise variance is normalized to $\sigma_{n}^{2}=1$. Channel gain variances are all unity $\left(\delta_{s, r}^{2}=\delta_{r, d}^{2}=\delta_{s, d}^{2}=1\right)$ except asymmetric cases.

In Fig. 3, the SER performance is compared for the ATRS and fixed threshold schemes, assuming two fixed thresholds that are computed at $P / \sigma_{n}^{2}=15 d B$ and $P / \sigma_{n}^{2}=25 d B$. We see that the latter shows similar performance only at their respective SNRs, but much worse performance than the former in other SNR range. This demonstrates that using adaptive threshold is prerequisite to achieve acceptable performance.

Fig. 4 shows that the best SER performance is achieved when a source has information about all instantaneous channel gains. As for the selection metric we have used the harmonic mean function of $S-R_{i}$ and $R_{i}-D$ instantaneous channel gains, defined as

$$
\max _{i} \frac{2 q_{1} q_{2} \beta_{r_{i}, d} \beta_{s, r_{i}}}{q_{1} \beta_{r_{i}, d}+q_{2} \beta_{s, r_{i}}}
$$




$$
\begin{aligned}
& \sum_{k=1}^{N} P_{e}\left(s, r_{i} \mid k\right) P_{e}(s, d) P(K=k)= \\
& N \sum_{i=0}^{N-1}\left(\begin{array}{c}
N-1 \\
i
\end{array}\right)(-1)^{i} e^{-(i+1) \beta_{t h} / \delta_{r, d}^{2}} F_{1}\left(\frac{b P_{1} \delta_{s, r}^{2}}{\sigma_{n}^{2} \sin ^{2} \theta}+i+1\right) F_{1}\left(\frac{b P_{1} \delta_{s, d}^{2}}{\sigma_{n}^{2} \sin ^{2} \theta}+1\right) \\
& \sum_{k=1}^{N} P_{e}\left(s, r_{i}, d \mid k\right) P(K=k)= \\
& \left(1-\left(1-e^{-\beta_{t h} / \delta_{r, d}^{2}}\right)^{N}\right) F_{1}\left(\left(\frac{b P_{1} \delta_{s, d}^{2}}{\sigma_{n}^{2} \sin ^{2} \theta}+1\right)\left(\frac{b P_{2} \delta_{r, d}^{2}}{\sigma_{n}^{2} \sin ^{2} \theta}+1\right) \exp \left(\frac{b P_{2} \beta_{t h}}{\sigma_{n}^{2} \sin ^{2} \theta}\right)\right) \\
& \sum_{k=1}^{N} P_{e}\left(s, r_{i} \mid k\right) P_{e}\left(s, r_{i}, d \mid k\right) P(K=k)= \\
& N \sum_{i=0}^{N-1}\left(\begin{array}{c}
N-1 \\
i
\end{array}\right)(-1)^{i} e^{-(i+1) \beta_{t h} / \delta_{r, d}^{2}} F_{1}\left(\frac{b P_{1} \delta_{s, r}^{2}}{\sigma_{n}^{2} \sin ^{2} \theta}+i+1\right) F_{1}\left(\left(\frac{b P_{1} \delta_{s, d}^{2}}{\sigma_{n}^{2} \sin ^{2} \theta}+1\right)\left(\frac{b P_{2} \delta_{r, d}^{2}}{\sigma_{n}^{2} \sin ^{2} \theta}+1\right) \exp \left(\frac{b P_{2} \beta_{t h}}{\sigma_{n}^{2} \sin ^{2} \theta}\right)\right) \\
& P_{e}\left(s, r_{i} \mid k=0\right) P_{e}(s, d) P(K=0)=\left(1-e^{-\beta_{t h} / \delta_{r, d}^{2}}\right)^{N} F_{1}\left(\frac{b P_{1} \delta_{s, r}^{2}}{\sigma_{n}^{2} \sin ^{2} \theta}+1\right) F_{1}\left(\frac{b P_{2} \delta_{s, d}^{2}}{\sigma_{n}^{2} \sin ^{2} \theta}+1\right) \\
& P_{e}\left(s, r_{i}, d \mid k=0\right) P(K=0)= \\
& \left(1-e^{-\beta_{t h} / \delta_{r, d}^{2}}\right)^{N-1} F_{1}\left(\left(\frac{b P_{1} \delta_{r, d}^{2}}{\sigma_{n}^{2} \sin ^{2} \theta}+1\right)\left(\frac{b P_{2} \delta_{s, d}^{2}}{\sigma_{n}^{2} \sin ^{2} \theta}+1\right)\left(1-\exp \left(-\frac{b P_{2} \beta_{t h}}{\sigma_{n}^{2} \sin ^{2} \theta}-\frac{\beta_{t h}}{\delta_{r, d}^{2}}\right)\right)^{-1}\right) \\
& P_{e}\left(s, r_{i} \mid k=0\right) P_{e}\left(s, r_{i}, d \mid k=0\right) P(K=0)= \\
& \left(1-e^{-\beta_{t h} / \delta_{r, d}^{2}}\right)^{N-1} F_{1}\left(\frac{b P_{1} \delta_{s, d}^{2}}{\sigma_{n}^{2} \sin _{\theta}^{2}}+1\right) F_{1}\left(\left(\frac{b P_{1} \delta_{r, d}^{2}}{\sigma_{n}^{2} \sin ^{2} \theta}+1\right)\left(\frac{b P_{2} \delta_{s, d}^{2}}{\sigma_{n}^{2} \sin ^{2} \theta}+1\right)\left(1-\exp \left(-\frac{b P_{2} \beta_{t h}}{\sigma_{n}^{2} \sin ^{2} \theta}-\frac{\beta_{t h}}{\delta_{r, d}^{2}}\right)\right)^{-1}\right) \\
& P_{e} \leq\left(\frac{M-1}{M}\right) \frac{\sigma_{n}^{2}}{b P_{1} \delta_{s, d}^{2}+\sigma_{n}^{2}} \frac{\sigma_{n}^{2}}{b P_{2} \delta_{r, d}^{2}+\sigma_{n}^{2}}\left(\left(1-e^{-\beta_{t h} / \delta_{r, d}^{2}}\right)^{N-1}\left(1-e^{-b_{s p k} P_{2} \beta_{t h} / \sigma_{n}^{2}}\right)+e^{-b_{s p k} P_{2} \beta_{t h} / \sigma_{n}^{2}}\right) \\
& +\left(\frac{M-1}{M}\right)^{2} \frac{\sigma_{n}^{2}}{b P_{1} \delta_{s, d}^{2}+\sigma_{n}^{2}} \frac{\sigma_{n}^{2}}{b P_{1} \delta_{s, r}^{2}+\sigma_{n}^{2}}\left(\left(1-e^{-\beta_{t h} / \delta_{r, d}^{2}}\right)^{N-1}\left(1+(N-1) e^{-\beta_{t h} / \delta_{r, d}^{2}}\right)\right.
\end{aligned}
$$

where the constants $q_{1}$ and $q_{2}$ are found in [6]. It should be noted that the SER performance of the proposed ATRS scheme nearly approaches the best one. Meanwhile, the worst performance occurs when the source knows $R_{i}-D$ average channel gain $\delta_{r_{i}, d}^{2}$ instead of instantaneous channel gain $\beta_{r_{i}, d}$ where (26) is used as the selection metric. Here, the ATRS scheme provides much better SER performance than that of using $R_{i}-D$ average channel gain, though both schemes achieve the same diversity order of two. Besides, 'harmonic mean threshold feedback' uses the harmonic mean as threshold metric instead of instantaneous channel gain $\beta_{r_{i}, d}$, which performs close to the ATRS scheme. But the ATRS scheme is better than 'harmonic mean threshold feedback' in view of channel information, since the source can know that $R_{i}-D$ channel gain is higher than threshold, whereas $R_{i}-D$ channel gain is vague if the harmonic mean is used instead.
In Fig. 5, the SER performance improves as the number of relays increases. Exact and upper bound on the SER, and the simulation result are all well matched. In multiple relay network the ATRS scheme is shown to provide acceptable performance in most operating SNR range though full diversity order is not achieved.

Fig. 6 shows the SER performance under asymmetric link conditions. Here, average channel gains of $S-R$ links are $\delta_{s, r_{1}}^{2}=0.5, \delta_{s, r_{2}}^{2}=1.5, \delta_{s, r_{3}}^{2}=1, \delta_{s, r_{4}}^{2}=1$, whereas for $R$ $D$ links $\delta_{r_{1}, d}^{2}=1.5, \delta_{r_{2}, d}^{2}=0.5, \delta_{r_{3}, d}^{2}=1, \delta_{r_{4}, d}^{2}=1$. Note that mean of $R-D$ and $S-R$ average channel gains is same (unity) for both symmetric and asymmetric cases. We have assumed the same adaptive threshold for which upper bounds are well matched with the corresponding simulation results. When compared with symmetric case in Fig. 5, asymmetric case nearly approaches the symmetric case. 


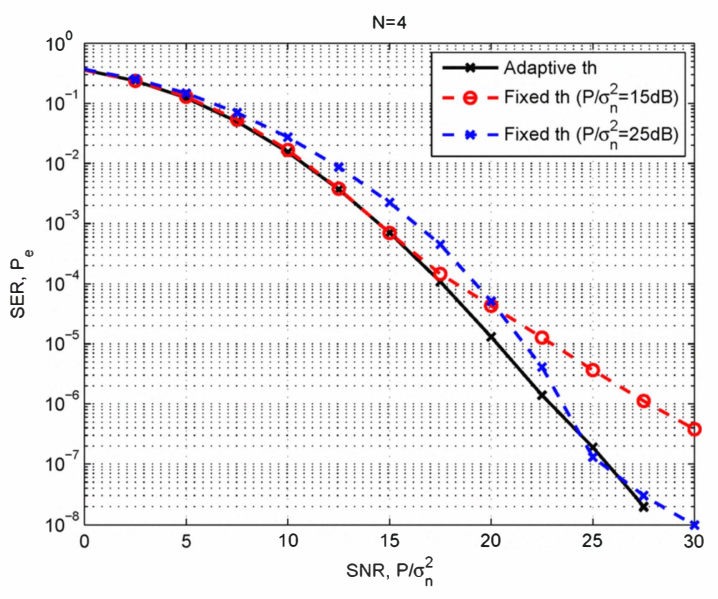

Fig. 3. SER comparison with adaptive threshold and two fixed thresholds at $P / \sigma_{n}^{2}=15 d B$ and $P / \sigma_{n}^{2}=25 d B$ when $N=4$.

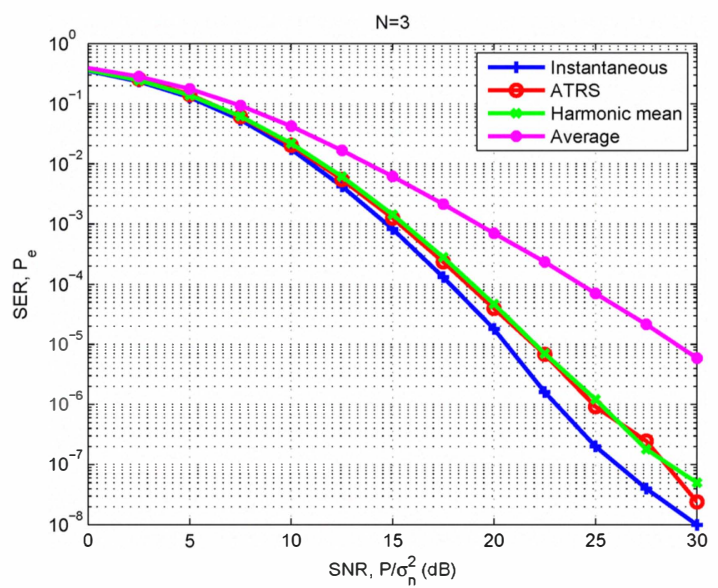

Fig. 4. SER comparison with varying degree of accuracy of $R-D$ link channel gain information when $N=3$.

\section{CONCLUSION}

We have proposed adaptive threshold based relay selection for minimum feedback in DF multiple relay network, which aims to maintain acceptable SER performance with minimum feedback. Exact and upper bound on the SER expression were derived whose theoretical results have been validated through simulation. Adaptive threshold was found from the upper bound for a simple calculation while the SER being minimized. Though full diversity order is not achieved, i.e., down to 2, the adaptive threshold scheme gives a competitive performance in practical SNR range, compared to the ideal case that a source knows all channel gains. It was shown that using fixed threshold causes severe degradation of the SER, and upper bound and simulation result are well matched under asymmetric link conditions.

\section{ACKNOWLEDGMENT}

This research was supported in part by the MKE (Ministry of Knowledge Economy), Korea, under the ITRC (Information Technology Research Center) support program supervised by

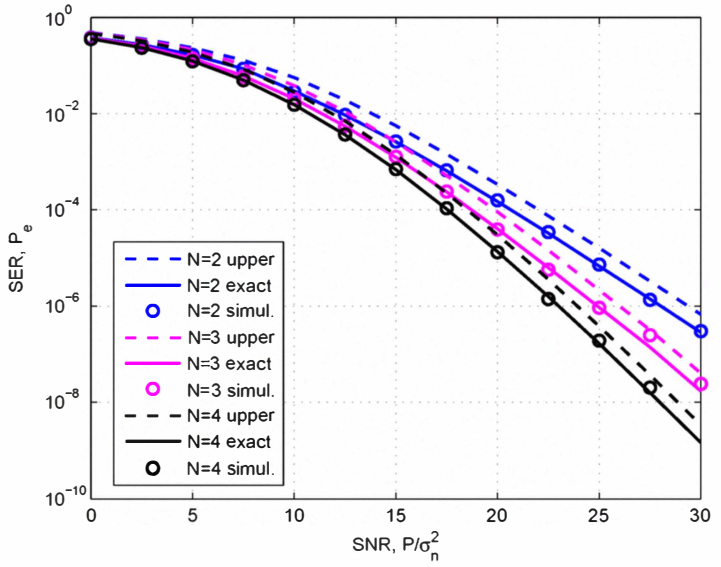

Fig. 5. The SER simulated with adaptive threshold, exact and upper bound curves plotted under symmetric link conditions.

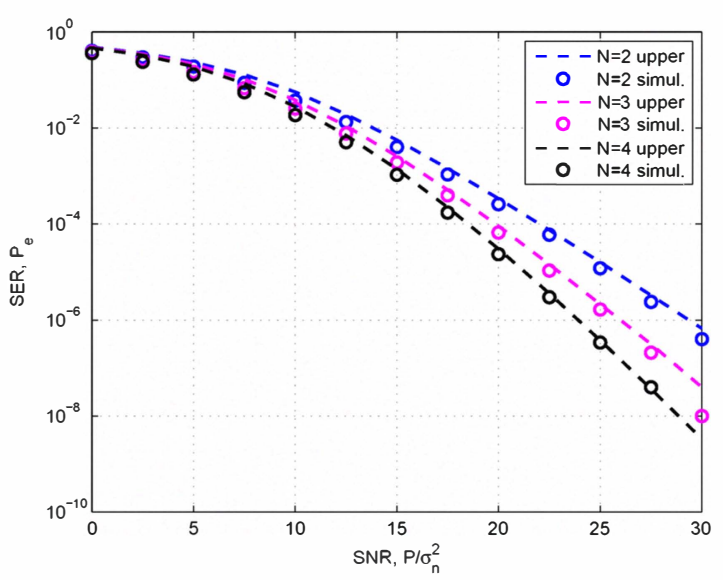

Fig. 6. The SER simulated with adaptive threshold, upper bound curve plotted under asymmetric link conditions.

the NIPA (National IT Industry Promotion Agency (NIPA2010-(C1090-1011-0005)) and in part by Samsung Electronics, Inc.

\section{REFERENCES}

[1] A. Sendonaris, E. Erkip, and B. Aazhang, "User cooperation diversity Part I and Part II," IEEE Trans. Commun., vol. 51, pp. 1927-1948, Nov. 2003.

[2] J. N. Laneman, G. W. Wornell, and D. N. C. Tse, "An efficient protocol for realizing cooperative diversity in wireless networks," Proc. IEEE ISIT '01, pp. 294, Washington D.C., June 2001.

[3] T. E. Hunter and A. Nosratinia, "Cooperative diversity through coding," Proc. IEEE ISIT '02, pp. 220, Laussane, Switzerland, July 2002.

[4] M. O. Hasna and M. S. Alouini, "Perfomance analysis of two-hop relayed transmissions over Rayleigh fading channel," Proc. IEEEE VTC '02 Fall, vol. 4, pp. 1992-1996, Sept. 2002.

[5] W. S. Su, A. K. Sadek, and K. J. R. Liu, "SER performance analysis and optimum power allocation for decode and forward cooperation procotol in wireless network," Proc. IEEE WCNC '05, vol. 2, pp. 984-989, Mar. 2005.

[6] A. S. Ibrahim, A. K. Sadek, W. Su, and K. J. R. Liu, "Cooperative communication with relay-selection: When to cooperate and whom to cooperate with?," IEEE Trans. Wireless Commun., vol. 7, pp. 2814-2827, July 2008.

[7] M. K. Simon, M. S. Alouini, Digital Communication over Fading Channels. Wiley-Interscience, 2nd ed. 2004. 